June 19, 2003

hep-th/0306193

EFI-03-23

\title{
The UV/IR Interplay in Theories with Space-time Varying Non-commutativity
}

\author{
Daniel Robbins ${ }^{1}$ and Savdeep Sethi ${ }^{2}$ \\ Enrico Fermi Institute, University of Chicago, Chicago, IL 60637, USA
}

\begin{abstract}
We consider scalar field theory with space and space-time-dependent non-commutativity. In perturbation theory, we find that the structure of the UV/IR mixing is quite different from cases with constant non-commutativity. In particular, UV/IR mixing becomes intertwined in an interesting way with violations of momentum conservation.
\end{abstract}

\footnotetext{
${ }^{1}$ email: robbins@theory.uchicago.edu

${ }^{2}$ email: sethi@theory.uchicago.edu
} 


\section{Introduction}

Recently there have been several examples, coming from string theory, of non-commutative structure with a space-time-dependent noncommutativity parameter [1,2,3,4,5,6]. In many of these examples, the non-commuting directions in space-time form a nilpotent Lie algebra:

$$
\left[\hat{x}^{\mu}, \hat{x}^{\nu}\right]=i \theta_{\rho}^{\mu \nu} \hat{x}^{\rho}
$$

where the structure constants $\theta_{\rho}^{\mu \nu}$ really are constant. We will use hats to denote noncommuting coordinates, and unhatted variables for their commutative counterparts. This algebra is very special because the non-commutativity depends only linearly on the spacetime coordinates.

This kind of non-commutative space-time has been considered before; see, for example, [7, 8, 9, 10]. One can use a Weyl quantization procedure to write down a GroenewaldMoyal star product [8, 11]. To write the explicit form of the star-product requires the Baker-Campbell-Hausdorff formula. The examples that interest us will have either all double commutators or all triple commutators vanishing, and we can write explicitly

$$
f * g(x)=\left.e^{\frac{i}{2} \theta_{\rho}^{\mu \nu} x^{\rho} \partial_{\mu}^{(1)} \partial_{\nu}^{(2)}} f\left(x_{1}\right) g\left(x_{2}\right)\right|_{x_{1}=x_{2}=x},
$$

when all double commutators vanish, or

$$
f * g(x)=\left.e^{\frac{i}{2} \theta_{\rho}^{\mu \nu} x^{\rho} \partial_{\mu}^{(1)} \partial_{\nu}^{(2)}+\frac{1}{12} \theta_{\lambda}^{\mu \nu} \theta_{\rho}^{\lambda \sigma} x^{\rho} \partial_{\mu}^{(1)} \partial_{\nu}^{(2)}\left(\partial_{\sigma}^{(1)}-\partial_{\sigma}^{(2)}\right)} f\left(x_{1}\right) g\left(x_{2}\right)\right|_{x_{1}=x_{2}=x}
$$

when all triple commutators vanish.

This product is automatically associative because the associativity condition on the product (for general $\theta^{\mu \nu}$ parameters)

$$
\theta^{\mu \nu} \partial_{\nu} \theta^{\rho \sigma}+\theta^{\rho \nu} \partial_{\nu} \theta^{\sigma \mu}+\theta^{\sigma \nu} \partial_{\nu} \theta^{\mu \rho}=0
$$

becomes simply the Jacobi identity on the structure coefficients

$$
\theta_{\lambda}^{\mu \nu} \theta_{\nu}^{\rho \sigma}+\theta_{\lambda}^{\rho \nu} \theta_{\nu}^{\sigma \mu}+\theta_{\lambda}^{\sigma \nu} \theta_{\nu}^{\mu \rho}=0
$$

which is guaranteed by the Lie algebra structure (11).

Quantum field theories on these non-commutative spaces are quite fascinating, and largely unexplored (though see [9] for a similar study on another Lie algebra example). Our aim in this work is to study the perturbative structure of these theories along the 
lines of [12,13]. As we shall see, the physics is quite different from theories with constant non-commutativity. We will consider scalar field theory. We will show that space-timedependent non-commutativity explicitly breaks momentum conservation in a way that becomes entwined with UV/IR mixing. We would like to interpret our results using a 'dipolelike' explanation which proved useful in the case of constant non-commutativity [14, 15]. However, it seems likely that such an explanation will involve strings rather than particles since (in a stringy context) $H=d B$ is non-zero. This, in turn, may require an analysis along the lines described in [16].

There are many directions to explore: a basic issue is the perturbative consistency of these theories. It should be possible to address this issue using the techniques of [17]. When the non-commutativity parameter depends on time, the resulting non-local theory is quite unusual (even if the parameter is purely spatial). Understanding the conditions under which such theories make sense is important. The case of Yang-Mills theory built from a space-time varying product is also interesting, but more subtle even in its classical definition. Yang-Mills theories with space-time varying non-commutativity appear in holographic descriptions of cosmological space-times [1], and have also been considered recently for phenomenology [18].

Note added: After we had completed this project, we received an interesting paper [19] which contains related observations.

\section{Two Particular Examples}

We will focus on two examples that are both realized in string theory.

\subsection{A space-dependent case}

The first example is from [5], and is realized in massive type IIA. The structure constants are,

$$
\theta_{3}^{12}=-\theta_{3}^{21}=\alpha
$$

corresponding to a non-commutative space with relations

$$
\left[\hat{x}^{1}, \hat{x}^{2}\right]=i \alpha \hat{x}^{3}, \quad\left[\hat{x}^{1}, \hat{x}^{3}\right]=\left[\hat{x}^{2}, \hat{x}^{3}\right]=0
$$


The star product of several functions is given by

$$
f_{1} * f_{2} * \cdots * f_{n}=e^{\frac{i}{2} \alpha x^{3} \sum_{a<b}\left(\partial_{1}^{(a)} \partial_{2}^{(b)}-\partial_{2}^{(a)} \partial_{1}^{(b)}\right)} f_{1} f_{2} \cdots f_{n}
$$

An important property satisfied by this product is the relation

$$
f * g=f g+\text { total divergence. }
$$

In this case, this is true for the same reason as in the case of the standard star product. Namely, consider a term in the integrated product

$$
\left.\int \frac{1}{n !}\left\{\frac{i}{2} \theta_{\rho}^{\mu \nu} x^{\rho} \partial_{\mu}^{(1)} \partial_{\nu}^{(2)}\right\}^{n} f\left(x_{1}\right) g\left(x_{2}\right)\right|_{x_{1}=x_{2}=x} .
$$

With the choice (6), we can freely integrate by parts to move one $\partial_{\mu}$ from $f$ to $g$. This gives zero up to a total divergence. This relation means that the tree-level propagator for an action involving this star product will agree with the usual commutative case. Note that because of the explicit $x^{3}$-dependence in the product, the momentum charge $P^{3}$ is not conserved.

\subsection{A space-time-dependent case}

In the case of the algebra coming from study of the null-brane quotient [20, originally described in [1, the structure constants are

$$
\theta_{+}^{x z}=-\theta_{+}^{z x}=\theta_{x}^{-z}=-\theta_{x}^{z-}=\tilde{R}
$$

which corresponds to a non-commutative space-time with non-vanishing relations

$$
[\hat{x}, \hat{z}]=i \tilde{R} \hat{x}^{+}, \quad\left[\hat{x}^{-}, \hat{z}\right]=i \tilde{R} \hat{x}
$$

As derived in [6], this algebra leads to a closed form Groenewald-Moyal star product between functions of the commutative variables

$$
\begin{aligned}
f_{1} * f_{2}= & \exp \left[\frac{i}{2} \theta_{\rho}^{\mu \nu} x^{\rho} \partial_{\mu}^{(1)} \partial_{\nu}^{(2)}-\frac{1}{12} \theta_{\rho}^{\mu \nu} \theta_{\nu}^{\sigma \lambda} x^{\rho} \partial_{\sigma}^{(1)} \partial_{\lambda}^{(2)}\left(\partial_{\mu}^{(1)}-\partial_{\mu}^{(2)}\right)\right] f_{1} f_{2} \\
= & \exp \left[\frac{i}{2} \tilde{R} x^{+}\left(\partial_{x}^{(1)} \partial_{z}^{(2)}-\partial_{z}^{(1)} \partial_{x}^{(2)}\right)+\frac{i}{2} \tilde{R} x\left(\partial_{-}^{(1)} \partial_{z}^{(2)}-\partial_{z}^{(1)} \partial_{-}^{(2)}\right)\right. \\
& \left.+\frac{1}{12} \tilde{R}^{2} x^{+}\left(\partial_{-z}^{(1)} \partial_{z}^{(2)}-\partial_{z z}^{(1)} \partial_{-}^{(2)}-\partial_{-}^{(1)} \partial_{z z}^{(2)}+\partial_{z}^{(1)} \partial_{-z}^{(2)}\right)\right] f_{1} f_{2}
\end{aligned}
$$


Here $\partial^{(i)}$ is understood to act only on $f_{i}$ (so in particular no derivatives act on the coordinates in the exponent). It is less trivial to check that

$$
f * g=f g+\text { total derivative }
$$

in this case. To see this, we need to examine terms in the expansion of (13)

$$
\begin{aligned}
& \int \frac{1}{n !}\left[\frac{i}{2} \tilde{R} x^{+}\left(\partial_{x}^{(1)} \partial_{z}^{(2)}-\partial_{z}^{(1)} \partial_{x}^{(2)}\right)+\frac{i}{2} \tilde{R} x\left(\partial_{-}^{(1)} \partial_{z}^{(2)}-\partial_{z}^{(1)} \partial_{-}^{(2)}\right)\right. \\
& \left.+\frac{1}{12} \tilde{R}^{2} x^{+}\left(\partial_{-z}^{(1)} \partial_{z}^{(2)}-\partial_{z z}^{(1)} \partial_{-}^{(2)}-\partial_{-}^{(1)} \partial_{z z}^{(2)}+\partial_{z}^{(1)} \partial_{-z}^{(2)}\right)\right]^{n} f_{1} f_{2}
\end{aligned}
$$

There are no explicit factors of $z, x^{-}$in (15) so we can integrate by parts to make the triple product terms, and the double product terms proportional to $x$ vanish (up to total divergences). That leaves the first term in (15) which can now safely be integrated by parts to give zero. As in the previous example, quadratic terms in an action built from this star product reduce to those of a commutative theory.

At first glance, one might worry that the term in (13) that is quadratic in $\tilde{R}$ is not a phase, since there is no $i$ in front of it. However, if write the interaction in terms of $x^{3}$ and the $z, x^{-}$momenta then the partial derivatives are replaced by factors of $i k_{\mu}$, and all the terms become phases.

It is not very difficult to generalize the result (13) to a finite product $f_{1} * f_{2} * \cdots * f_{n}$. The result takes the deceptively simple form

$$
\hat{A} \hat{B} \hat{C} f_{1} \cdots f_{n}
$$

where $\hat{A}, \hat{B}, \hat{C}$ are mutually commuting differential operators taking the explicit forms

$$
\begin{aligned}
\hat{A} & =\prod_{a<b} \exp \left[\frac{i}{2} \tilde{R} x^{+}\left(\partial_{x}^{(a)} \partial_{z}^{(b)}-\partial_{z}^{(a)} \partial_{x}^{(b)}\right)+\frac{i}{2} \tilde{R} x\left(\partial_{-}^{(a)} \partial_{z}^{(b)}-\partial_{z}^{(a)} \partial_{-}^{(b)}\right)\right] \\
\hat{B} & =\prod_{a<b} \exp \left[\frac{1}{12} \tilde{R}^{2} x^{+}\left(\partial_{-z}^{(a)} \partial_{z}^{(b)}-\partial_{z z}^{(a)} \partial_{-}^{(b)}-\partial_{-}^{(a)} \partial_{z z}^{(b)}+\partial_{z}^{(a)} \partial_{-z}^{(b)}\right)\right] \\
\hat{C} & =\prod_{a<b<c} \exp \left[\frac{1}{6} \tilde{R}^{2} x^{+}\left(2 \partial_{z}^{(a)} \partial_{-}^{(b)} \partial_{z}^{(c)}-\partial_{-}^{(a)} \partial_{z}^{(b)} \partial_{z}^{(c)}-\partial_{z}^{(a)} \partial_{z}^{(b)} \partial_{-}^{(c)}\right)\right] .
\end{aligned}
$$

This gives us the modification to the vertex factor in $\phi^{n}$ scalar field theory in position space. Compare this with the usual constant $\theta$ case, where there is only an operator corresponding 
to $\hat{A}$, and it has no explicit position dependence. So in momentum space, it can simply be rewritten as a phase factor,

$$
V\left(k_{1}, \ldots, k_{n}\right)=\prod_{a<b} e^{-\frac{i}{2} \theta^{\mu \nu} k_{\mu}^{(a)} k_{\nu}^{(b)}}
$$

and the only modification to the momentum space Feynman rules is the inclusion of this factor at each vertex. Our situation is a little more complicated because of the appearance of both positions and derivatives in our phases. In particular, because of the $x$ and $x^{+}$dependence, interactions will not conserve the $P^{x}, P^{-}$momenta.

\section{Perturbation Theory for the Space-Dependent Case}

\section{$3.1 \quad$ Feynman rules}

We would now like to consider a scalar field theory Lagrangian constructed from the spacedependent product (8),

$$
\mathcal{L}=\frac{1}{2}\left(\partial_{\mu} \phi\right)^{2}+\frac{1}{2} m^{2} \phi^{2}+\frac{g^{2}}{n !} \underbrace{\phi * \phi * \cdots * \phi}_{\mathrm{n} \text { times }} .
$$

This theory is a little simpler than the time-dependent case, and so provides a good set-up for us to first study the effects of space-time varying non-commutativity. Since this theory is static, we can analytically continue to the Euclidean theory. Because of (9), the quadratic terms in the action involve only ordinary products. The Feynman rules can then be written down in the following mixed picture (i.e. with integrations both over the positions of the vertices and over the momenta of the internal lines). We will simplify these rules in a moment. The rules are:

1. For each external line, a factor $e^{-i p \cdot x}$.

2. For each internal line, a factor $\frac{1}{p^{2}+m^{2}+i \epsilon} e^{-i p \cdot(x-y)}$.

3. For each vertex, a factor $g^{2} e^{-\frac{i}{2} \alpha x^{3} \sum_{a<b}\left(p_{1}^{(a)} p_{2}^{(b)}-p_{2}^{(a)} p_{1}^{(b)}\right)}$.

4. An integration $\int d^{4} x$ over the position of each vertex.

5. An integration $\int \frac{d^{4} p}{(2 \pi)^{4}}$ over each internal momentum. 
We can perform the integrations over $x^{0}, x^{1}$, and $x^{2}$ to produce delta functions which enforce conservation of $p_{0}, p_{1}$, and $p_{2}$ at each vertex. We could also perform the integration over $x^{3}$ to produce a delta function of the form

$$
\delta\left(\sum_{a} p_{3}^{(a)}+\frac{\alpha}{2} \sum_{a<b}\left(p_{1}^{(a)} p_{2}^{(b)}-p_{2}^{(a)} p_{1}^{(b)}\right)\right) .
$$

This already makes clear the difference between this case and the case of constant noncommutativity. With constant non-commutativity, the analogue of (20) is a phase of the form

$$
\delta\left(\sum_{a} p_{3}^{(a)}\right) \exp \left\{i \frac{\alpha}{2} \sum_{a<b}\left(p_{1}^{(a)} p_{2}^{(b)}-p_{2}^{(a)} p_{1}^{(b)}\right)\right\},
$$

while for us, the phase is replaced by momentum non-conservation. However, for some purposes, we will find it more helpful to leave the $x^{3}$ integrations undone for now. Our simplified Feynman rules become:

1. For each internal line, a factor $\frac{1}{p^{2}+m^{2}+i \epsilon}$.

2. For each vertex, a factor

$$
\begin{aligned}
& g^{2} \exp \left\{-i x^{3}\left[\sum_{a} p_{3}^{(a)}+\frac{1}{2} \alpha \sum_{a<b}\left(p_{1}^{(a)} p_{2}^{(b)}-p_{2}^{(a)} p_{1}^{(b)}\right)\right]\right\} \times \\
& (2 \pi)^{3} \delta\left(\sum_{a} p_{0}^{(a)}\right) \delta\left(\sum_{a} p_{1}^{(a)}\right) \delta\left(\sum_{a} p_{2}^{(a)}\right) .
\end{aligned}
$$

3. An integration $\int d x^{3}$ over the $x^{3}$ position of each vertex.

4. An integration $\int \frac{d^{4} p}{(2 \pi)^{4}}$ over each internal momentum.

\section{$3.2 \phi^{4}$ in four dimensions}

Let us now specialize to the non-commutative $\phi^{4}$ theory in four dimensions. We will compute the one-loop corrections to the propagator of this theory. In conventional noncommutative field theory, the vertex modification (21) is invariant under cyclic permutation of the momenta [12]. This result relies only on momentum conservation in the non-commutative directions, and so it continues to hold for us.

The same comment applies to the decomposition into planar and non-planar diagrams, again because momenta in the non-commuting directions are conserved at each vertex. As 

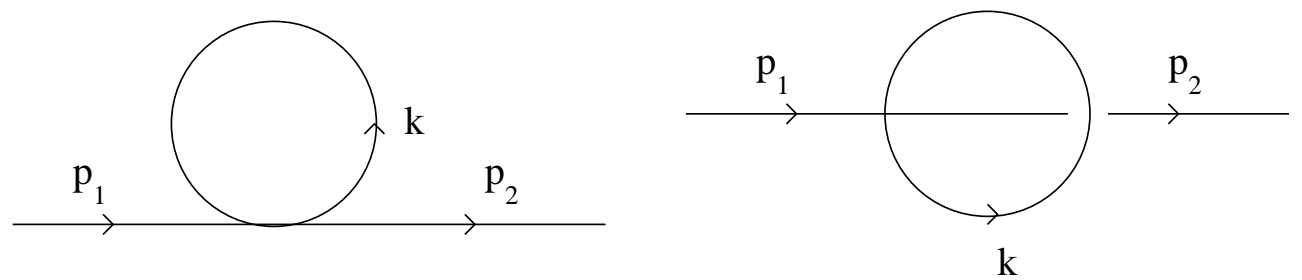

Figure 1: The planar and non-planar one-loop contributions to the $\phi^{4}$ propagator.

in conventional non-commutative $\phi^{4}$ theory, there are then two diagrams that contribute: one planar diagram and one non-planar diagram, both of which are depicted in Figure 1.

In the planar diagram, the extra phase factors at the vertex cancel and we have a contribution

$$
\begin{aligned}
\Gamma_{1 \text { planar }}^{(2)} & =\frac{g^{2}}{3(2 \pi)^{5}} \delta^{3}\left(p_{\perp}^{(1)}-p_{\perp}^{(2)}\right) \int d x^{3} \int \frac{d^{4} k}{k^{2}+m^{2}} e^{-i x^{3}\left(p_{3}^{(1)}-p_{3}^{(2)}\right)} \\
& =\frac{g^{2}}{3(2 \pi)^{4}} \delta^{4}\left(p^{(1)}-p^{(2)}\right) \int \frac{d^{4} k}{k^{2}+m^{2}},
\end{aligned}
$$

where $p_{\perp}$ represents the three conserved components of momentum, i.e. $p_{0}, p_{1}$, and $p_{2}$. This is exactly the same as in commutative $\phi^{4}$ theory up to a symmetry factor. This diagram is quadratically divergent.

The non-planar diagram is more interesting. Here the extra phase factors do not cancel and we get a contribution

$$
\Gamma_{1 \mathrm{np}}^{(2)}=\frac{g^{2}}{6(2 \pi)^{5}} \delta^{3}\left(p_{\perp}^{(1)}-p_{\perp}^{(2)}\right) \int d x^{3} \int \frac{d^{4} k}{k^{2}+m^{2}} e^{-i x^{3}\left\{p_{3}^{(1)}-p_{3}^{(2)}-\alpha\left(p_{1}^{(1)} k_{2}-p_{2}^{(1)} k_{1}\right)\right\}} .
$$

As usual for a massive theory, the only divergences come from the region of large $k$. IR divergences are supressed.

To examine this result more closely, we will rewrite the propagator using an integral over a Schwinger parameter

$$
\frac{1}{k^{2}+m^{2}}=\int_{0}^{\infty} d s e^{-s\left(k^{2}+m^{2}\right)} .
$$

The $k$ integrations then become Gaussians, and on performing those integrals, we obtain

$$
\begin{aligned}
\Gamma_{1 \text { planar }}^{(2)} & =\frac{g^{2}}{48 \pi^{2}} \delta^{4}\left(p^{(1)}-p^{(2)}\right) \int_{0}^{\infty} \frac{d s}{s^{2}} e^{-s m^{2}} \\
\Gamma_{1 \mathrm{np}}^{(2)} & =\frac{g^{2}}{192 \pi^{3}} \delta^{3}\left(p_{\perp}^{(1)}-p_{\perp}^{(2)}\right) \int_{0}^{\infty} \frac{d s}{s^{2}} \int d x e^{-s m^{2}-\frac{\alpha^{2}}{4 s} p_{n c}^{2}\left(x^{3}\right)^{2}-i x^{3} \Delta p_{3}} .
\end{aligned}
$$


We have used the abbreviations $p_{n c}^{2}=\left(p_{1}^{(1)}\right)^{2}+\left(p_{2}^{(1)}\right)^{2}$ and $\Delta p_{3}=p_{3}^{(1)}-p_{3}^{(2)}$ in the second integral. The large $k$ divergences have now become divergences at small values of $s$, so to regulate these integrals we include a multiplicative factor of $\exp \left[-1 /\left(\Lambda^{2} s\right)\right]$. This gives us

$$
\begin{aligned}
\Gamma_{1 \text { planar }}^{(2)} & =\frac{g^{2}}{48 \pi^{2}} \delta^{4}\left(p^{(1)}-p^{(2)}\right) \int_{0}^{\infty} \frac{d s}{s^{2}} e^{-s m^{2}-\frac{1}{\Lambda^{2} s}} \\
\Gamma_{1 \mathrm{np}}^{(2)} & =\frac{g^{2}}{192 \pi^{3}} \delta^{3}\left(p_{\perp}^{(1)}-p_{\perp}^{(2)}\right) \int_{0}^{\infty} \frac{d s}{s^{2}} \int d x^{3} e^{-s m^{2}-\frac{\alpha^{2}}{4 s} p_{n c}^{2}\left(x^{3}\right)^{2}-i x^{3} \Delta p_{3}-\frac{1}{\Lambda^{2} s}} .
\end{aligned}
$$

Since the $x^{3}$ integral in the non-planar amplitude above is now Gaussian, we will certainly be able to that integral, but first let us note a couple of features. For fixed values of $x^{3}$, the integral above consists of a phase factor times a standard Schwinger integral with an effective space-dependent cutoff

$$
\Lambda_{\text {eff }}^{2}=\frac{1}{1 / \Lambda^{2}+\alpha^{2}\left(x^{3}\right)^{2} p_{n c}^{2} / 4} .
$$

This is the same result that one would obtain from the noncommutative theory with constant $\theta^{12}=\alpha x^{3}$. Also, the fact that the $x^{3}$ integral is Gaussian implies the interaction is localized in the $x^{3}$ direction for $p_{n c}^{2} \neq 0$. The non-planar interaction is heavily supressed for large $\left|x^{3}\right|$.

We can see UV/IR mixing quite nicely from (27). If we first take the non-commutative momenta $p_{n c}^{2} \rightarrow 0$ then we can integrate over $x^{3}$ to get

$$
\delta\left(\Delta p_{3}\right)
$$

and a quadratically divergent contribution. In other words, we recover the commutative result. However, if $p_{n c}^{2} \neq 0$ then (setting $\Delta p_{3}=0$ for simplicity), we can integrate out $x^{3}$ to get a $s \rightarrow 0$ divergence

$$
\sim \int \frac{d s}{s^{3 / 2}}
$$

which is softer than the planar contribution. The order in which we take $p_{n c}^{2} \rightarrow 0$ and $\Lambda \rightarrow \infty$ matters just as in [13].

Let us be more precise. Performing the $x^{3}$ integration with $p_{n c}^{2} \neq 0$, we are left with

$$
\Gamma_{1 \mathrm{np}}^{(2)}=\frac{g^{2}}{192 \pi^{3}} \delta^{3}\left(p_{\perp}^{(1)}-p_{\perp}^{(2)}\right) \sqrt{\frac{4 \pi}{\alpha^{2} p_{n c}^{2}}} \int_{0}^{\infty} \frac{d s}{s^{3 / 2}} e^{-s m_{e f f}^{2}-\frac{1}{\Lambda^{2} s}},
$$

where we have now defined an effective mass

$$
m_{e f f}^{2}=m^{2}+\frac{\left(\Delta p_{3}\right)^{2}}{\alpha^{2} p_{n c}^{2}}
$$


Apart from the momentum-dependent factor out front, this is the type of Schwinger integral that we would evaluate for a three-dimensional theory. In fact, the integral can be evaluated exactly using the results listed in Appendix A. The result, along with the conventional planar contribution, is

$$
\begin{aligned}
\Gamma_{1 \text { planar }}^{(2)} & =\frac{g^{2}}{48 \pi^{2}} \delta^{4}\left(p^{(1)}-p^{(2)}\right)\left(\Lambda^{2}-m^{2} \ln \left(\frac{\Lambda^{2}}{m^{2}}\right)+O(1)\right) \\
\Gamma_{1 \mathrm{np}}^{(2)} & =\frac{g^{2}}{96 \pi^{2}} \delta^{3}\left(p_{\perp}^{(1)}-p_{\perp}^{(2)}\right) \frac{\Lambda}{\alpha \sqrt{p_{n c}^{2}}} e^{-2 \sqrt{m_{\text {eff }}^{2} / \Lambda^{2}}} .
\end{aligned}
$$

We can now compare this exact result for the non-planar amplitude (33) with our qualitative expectations. First, only the combination $\alpha^{2} p_{n c}^{2}$ appears. As $\alpha^{2} p_{n c}^{2} \rightarrow \infty$, i.e., either for infinite non-commutativity and generic momenta, or for finite non-commutativity and very large external momenta in the noncommuting directions, the non-planar amplitude is heavily suppressed relative to the planar amplitude. This is analogous to the large non-commutativity limit in theories with constant $\theta$. For finite non-zero $\alpha^{2} p_{n c}^{2}$, the UV divergence of the amplitude is softened from quadratic to linear in $\Lambda$ - the divergence that one would expect for $\phi^{4}$ theory in three dimensions. As we will see in the space-time noncommutative case, as $\theta$ depends on more coordinates, the UV divergence for the non-planar diagram becomes softer.

Finally, consider the limit $\alpha^{2} p_{n c}^{2} \rightarrow 0$. For $\Delta p_{3}$ nonzero, $m_{\text {eff }}^{2} \rightarrow 0$ and the exponential causes the amplitude to vanish. For $\Delta p_{3}=0$, the amplitude diverges. Indeed, since

$$
\int d\left(\Delta p_{3}\right) \frac{1}{\alpha \sqrt{p_{n c}^{2}}} e^{-2 \sqrt{m_{e f f}^{2} / \Lambda^{2}}}=\Lambda \int d u e^{-2 \sqrt{u^{2}+m^{2} / \Lambda^{2}}}<\infty
$$

it follows that in this limit the non-planar amplitude is simply proportional to $\delta\left(\Delta p_{3}\right)$ as we expect:

$$
\begin{aligned}
\lim _{\alpha^{2} p_{n c}^{2} \rightarrow 0} \Gamma_{1 \mathrm{np}}^{(2)} & =\frac{g^{2}}{96 \pi^{2}} \delta^{4}\left(p^{(1)}-p^{(2)}\right) \Lambda^{2} \int d u e^{-2 \sqrt{u^{2}+m^{2} / \Lambda^{2}}} \\
& \simeq \frac{g^{2}}{96 \pi^{2}} \delta^{4}\left(p^{(1)}-p^{(2)}\right)\left(\Lambda^{2}-m^{2} \ln \left(\frac{\Lambda^{2}}{m^{2}}\right)+O(1)\right)
\end{aligned}
$$

So in this limit the correct commutative behavior is recovered. Note also that for finite $\alpha$ there are always external momenta $\left(p_{1}, p_{2} \approx 1 / \alpha\right)$ for which the non-planar contribution is non-neglible when compared to the planar diagram. For completeness, we include in Appendix B the analogous 1-loop computation for $\phi^{3}$ theory in six dimensions. 


\section{The Space-time-dependent Case}

We now turn to the algebra of section (2.2) derived from the null-brane quotient. We can again write down Feynman rules for $\phi^{4}$ theory in four dimensions defined using the star-product (13). The Minkowski space rules are

1. For each internal line, a factor $\frac{-i}{p^{2}+m^{2}+i \epsilon}$.

2. For each vertex, a factor

$$
\begin{array}{r}
g^{2} \exp \left\{-i x\left[\sum_{a} p_{x}^{(a)}+\frac{1}{2} \tilde{R} \sum_{a<b}\left(p_{-}^{(a)} p_{z}^{(b)}-p_{z}^{(a)} p_{-}^{(b)}\right)\right]-i x^{+}\left[\sum_{a} p_{+}^{(a)}\right.\right. \\
\left.\left.+\frac{1}{2} \tilde{R} \sum_{a<b}\left(p_{x}^{(a)} p_{z}^{(b)}-p_{z}^{(a)} p_{x}^{(b)}\right)-\frac{\tilde{R}^{2}}{6} \sum_{a<b<c}\left(2 p_{z}^{(a)} p_{-}^{(b)} p_{z}^{(c)}-p_{-}^{(a)} p_{z}^{(b)} p_{z}^{(c)}-p_{z}^{(a)} p_{z}^{(b)} p_{-}^{(c)}\right)\right]\right\} \\
\times(2 \pi)^{2} \delta\left(\sum_{a} p_{-}^{(a)}\right) \delta\left(\sum_{a} p_{z}^{(a)}\right) .
\end{array}
$$

3. An integration $\int d x^{+} d x$ over the position of each vertex.

4. An integration $\int \frac{d^{4} p}{(2 \pi)^{4}}$ over each internal momentum.

Notice that the vertex factor contribution that would have come from $\hat{B}$ in (17) is absent. One can check that it in fact vanishes by conservation of $p_{-}$and $p_{z}$.

Let us again consider the one-loop correction to the propagator. As before we have contributions from the same two diagrams displayed in figure 1. For the planar diagram, the extra vertex factors cancel, and we are again left with the same contribution as in the commutative case.

The non-planar contribution is now given by

$$
\begin{aligned}
& \Gamma_{1 \mathrm{np}}^{(2)}=\frac{g^{2}}{6(2 \pi)^{6}} \delta\left(p_{-}^{(1)}-p_{-}^{(2)}\right) \delta\left(p_{z}^{(1)}-p_{z}^{(2)}\right) \int d x^{+} d x \int d^{4} k \frac{-i}{k^{2}+m^{2}+i \epsilon} \\
& \times \exp \left[-i x\left(\Delta p_{x}+\tilde{R}\left(p_{z} k_{-}-p_{-} k_{z}\right)\right)-i x^{+}\left(\Delta p_{+}+\tilde{R}\left(p_{z} k_{x}-p_{x}^{(2)} k_{z}-\frac{1}{2} p_{z} \Delta p_{x}\right)\right.\right. \\
& \left.\left.-\frac{1}{2} \tilde{R}^{2}\left(p_{z} p_{z} k_{-}-p_{z} p_{-} k_{z}-p_{z} k_{z} k_{-}+p_{-} k_{z} k_{z}\right)\right)\right]
\end{aligned}
$$

Because the (bare) propagator is unmodified, we see that the pole structure in the above integrals is the same as the usual case. This makes it plausible that we can rotate the $k_{0}$ contour, replacing $k_{0}$ with $i \tilde{k}_{0}$ (here we are using $k_{ \pm}=\left(k_{0} \pm k_{1}\right) / \sqrt{2}$ ). If we go ahead and 
make this change, and then replace the propagator with a Schwinger integral, we obtain (for simplicity, we drop the tildes on $k_{0}$ )

$$
\begin{aligned}
\Gamma_{1 \mathrm{np}}^{(2)}= & \frac{g^{2}}{6(2 \pi)^{6}} \delta\left(p_{-}^{(1)}-p_{-}^{(2)}\right) \delta\left(p_{z}^{(1)}-p_{z}^{(2)}\right) \int_{0}^{\infty} d s \int d x^{+} d x d^{4} k \exp \left[-s\left(k^{2}+m^{2}\right)\right. \\
& -i x\left(\Delta p_{x}-\tilde{R} p_{-} k_{z}-\frac{1}{\sqrt{2}} \tilde{R} p_{z} k_{1}\right)-i x^{+}\left(\Delta p_{+}+\tilde{R}\left(p_{z} k_{x}-p_{x}^{(2)} k_{z}-\frac{1}{2} p_{z} \Delta p_{x}\right)\right. \\
& \left.+\frac{1}{2} \tilde{R}^{2}\left(\frac{1}{\sqrt{2}} p_{z} p_{z} k_{1}+p_{z} p_{-} k_{z}-\frac{1}{\sqrt{2}} p_{z} k_{z} k_{1}-p_{-} k_{z} k_{z}\right)\right) \\
& \left.+\frac{1}{\sqrt{2}} x \tilde{R} p_{z} k_{0}-\frac{1}{2 \sqrt{2}} x^{+} \tilde{R}^{2} p_{z}\left(p_{z}-k_{z}\right) k_{0}\right] .
\end{aligned}
$$

The momentum integrals are now Gaussian. Performing them, we obtain

$$
\begin{aligned}
\Gamma_{1 \mathrm{np}}^{(2)}= & \frac{g^{2}}{3 \cdot 2^{7} \pi^{4}} \delta\left(p_{-}^{(1)}-p_{-}^{(2)}\right) \delta\left(p_{z}^{(1)}-p_{z}^{(2)}\right) \int_{0}^{\infty} d s \int d x^{+} d x \frac{1}{s^{3 / 2} B^{1 / 2}} \\
& \times \exp \left[-s m^{2}-i x \Delta p_{x}-i x^{+}\left(\Delta p_{+}-\frac{1}{2} \tilde{R} p_{z} \Delta p_{x}\right)-\frac{\tilde{R}^{2} p_{z}^{2}}{4 s}\left(x^{+}\right)^{2}\right. \\
& \left.-\frac{\tilde{R}^{2} p_{-}^{2}}{4 B}\left(x+\frac{p_{x}^{(2)}}{p_{-}} x^{+}-\frac{1}{2} \tilde{R} p_{z} x^{+}\right)^{2}\right]
\end{aligned}
$$

where we have defined $B=s-\frac{i}{2} \tilde{R}^{2} p_{-} x^{+}$.

If we now perform the integral over $x$, followed by the integral over $x^{+}$, we obtain

$$
\begin{aligned}
\Gamma_{1 \mathrm{np}}^{(2)} & =\frac{g^{2}}{96 \pi^{3}} \delta\left(p_{-}^{(1)}-p_{-}^{(2)}\right) \delta\left(p_{z}^{(1)}-p_{z}^{(2)}\right) \frac{1}{\tilde{R}^{2}\left|p_{-} p_{z}\right|} \int_{0}^{\infty} \frac{d s}{s} e^{-s m_{e f f}^{2}-\frac{1}{s \Lambda^{2}}} \\
& =\frac{g^{2}}{48 \pi^{3}} \delta\left(\Delta p_{-}\right) \delta\left(\Delta p_{z}\right) \frac{1}{\tilde{R}^{2}\left|p_{-} p_{z}\right|} K_{0}\left(2 \frac{m_{\text {eff }}}{\Lambda}\right),
\end{aligned}
$$

where now

$$
m_{e f f}^{2}=m^{2}+\frac{\left(\Delta p_{x}\right)^{2}}{\tilde{R}^{2} p_{-}^{2}}+\frac{1}{\tilde{R}^{2} p_{z}^{2}}\left(\Delta p_{+}-\frac{\bar{p}_{x} \Delta p_{x}}{p_{-}}\right)^{2},
$$

and $\bar{p}_{x}=\frac{1}{2}\left(p_{x}^{(1)}+p_{x}^{(2)}\right) . K_{0}$ is a modified Bessel function of the second kind.

For nonzero $\tilde{R}, p_{-}$, and $p_{z}$, the large $\Lambda$ behaviour is

$$
\Gamma_{1 \mathrm{np}}^{(2)}=\frac{g^{2}}{96 \pi^{3}} \delta\left(p_{-}^{(1)}-p_{-}^{(2)}\right) \delta\left(p_{z}^{(1)}-p_{z}^{(2)}\right) \frac{1}{\tilde{R}^{2}\left|p_{-} p_{z}\right|} \ln \left(\frac{\Lambda}{m_{e f f}}\right) .
$$

So for nonzero $\tilde{R}, p_{-}$, and $p_{z}$, the expected UV divergence is softened from quadratic to logarithmic. We should also note that in the limit $\tilde{R} \rightarrow \infty$, the non-planar amplitude is again suppressed (for generic momenta) relative to the planar amplitude. 
Finally, let us consider the behaviour for IR values of the external momenta. It turns out that as we take either $p_{z} \rightarrow 0$ or $p_{-} \rightarrow 0$, we restore one delta function, while if we take $\tilde{R} \rightarrow 0$ (or equivalently both $p_{-} \rightarrow 0$ and $p_{z} \rightarrow 0$ at once) we restore the commutative limit and full momentum conservation. Explicitly,

$$
\begin{aligned}
\lim _{p_{z} \rightarrow 0} \Gamma_{1 \mathrm{np}}^{(2)} & =\frac{g^{2}}{96 \pi^{2}} \delta\left(\Delta p_{-}\right) \delta\left(\Delta p_{z}\right) \delta\left(\Delta p_{+}-\frac{\bar{p}_{x} \Delta p_{x}}{p_{-}}\right) \frac{\Lambda}{\tilde{R}\left|p_{-}\right|} e^{-\frac{2}{\Lambda} \sqrt{m^{2}+\frac{\left(\Delta p_{x}\right)^{2}}{\tilde{R}^{2} p_{-}^{2}}}} \\
\lim _{p_{-} \rightarrow 0} \Gamma_{1 \mathrm{np}}^{(2)} & =\frac{g^{2}}{96 \pi^{2}} \delta\left(\Delta p_{-}\right) \delta\left(\Delta p_{z}\right) \delta\left(\Delta p_{x}\right) \frac{\Lambda}{\tilde{R} \sqrt{p_{z}^{2}+\bar{p}_{x}^{2}}} e^{-\frac{2}{\Lambda} \sqrt{m^{2}+\frac{\left(\Delta p_{+}\right)^{2}}{\tilde{R}^{2}\left(p_{z}^{2}+\bar{p}_{x}^{2}\right)}}} \\
\lim _{\tilde{R} \rightarrow 0} \Gamma_{1 \mathrm{np}}^{(2)} & =\frac{g^{2}}{48 \pi^{2}} \delta^{4}(\Delta p) m \Lambda K_{1}\left(2 \frac{m}{\Lambda}\right)
\end{aligned}
$$

We should stress, in closing, that this theory is quite unusual. Energy and momentum are not conserved, and as a consequence, we expect to observe strange decay phenomena. However, the existence of strange theories of this kind is suggested by the behavior of strings in cosmological backgrounds.

\section{Acknowledgements}

We would like to thank Aki Hashimoto and Vikram Divvuri for helpful discussions. S. S. would like to thank the organizers of the 2003 Amsterdam Workshop on String Theory and Quantum Gravity for their hospitality. The work of D. R. is supported in part by a Julie Payette-NSERC PGS B Research Scholarship, and by NSF CAREER Grant No. PHY0094328. The work of S. S. is supported in part by NSF CAREER Grant No. PHY-0094328, and by the Alfred P. Sloan Foundation.

\section{A Some Useful Integrals}

We note that most of the integrals over Schwinger parameters that we need to perform give modified Bessel functions of the second kind, since

$$
I_{\mu}(m, \Lambda) \equiv \int_{0}^{\infty} d s s^{-\mu} \exp \left[-s m^{2}-\frac{1}{s \Lambda^{2}}\right]=2(m \Lambda)^{\mu-1} K_{\mu-1}\left(2 \frac{m}{\Lambda}\right) .
$$


Specifically, we need

$$
\begin{aligned}
I_{2}(m, \Lambda) & =2 m \Lambda K_{1}\left(2 \frac{m}{\Lambda}\right) \simeq \Lambda^{2}-m^{2} \ln \left(\frac{\Lambda^{2}}{m^{2}}\right) \\
I_{3 / 2}(m, \Lambda) & =2 \sqrt{m \Lambda} K_{1 / 2}\left(2 \frac{m}{\Lambda}\right)=\sqrt{\pi} \Lambda e^{-2 \frac{m}{\Lambda}} \\
I_{1}(m, \Lambda) & =2 K_{0}\left(2 \frac{m}{\Lambda}\right) \simeq \frac{1}{2} \ln \left(\frac{\Lambda}{m}\right) .
\end{aligned}
$$

We have included the exact closed form for $K_{1 / 2}$, and the large $\Lambda$ expansions for $K_{0}$ and $K_{1}$.

\section{B The One-loop Propagator in Six Dimensions}

We can repeat the one-loop analysis for $\phi^{4}$ theory in the case of $\phi^{3}$ theory in six dimensions. The Feynman rules are basically the same, and we again have one planar and one non-planar diagram to compute.

The loop diagrams involve two interaction vertices so we need to introduce two Schwinger parameters. Since the computations are straightforward, we will only display the results in terms of these parameters.

$$
\begin{aligned}
\Gamma_{1 \text { planar }}^{(2)}= & \frac{g^{4}}{256 \pi^{3}} \delta^{6}\left(p^{(1)}-p^{(2)}\right) \int d s_{1} d s_{2} \frac{1}{\left(s_{1}+s_{2}\right)^{2}} \frac{1}{\sqrt{\left(s_{1}+s_{2}\right)^{2}+\frac{1}{4} s_{1} s_{2} \alpha^{2} p_{n c}^{2}}} \\
& \times \exp \left[-\left(s_{1}+s_{2}\right)^{2} m^{2}-\frac{s_{1} s_{2}}{s_{1}+s_{2}} p_{\perp}^{2}-\frac{s_{1} s_{2}}{\left.s_{1}+s_{2}+\frac{s_{1} s_{2} \alpha^{2} p_{n c}^{2}}{4\left(s_{1}+s_{2}\right)} p_{3}^{2}\right]}\right. \\
\Gamma_{1 \mathrm{np}}^{(2)}= & \frac{g^{4}}{256 \pi^{7 / 2}} \delta^{5}\left(p_{\perp}^{(1)}-p_{\perp}^{(2)}\right) \frac{1}{\alpha \sqrt{p_{n c}^{2}}} \int d s_{1} d s_{2} \frac{1}{\left(s_{1}+s_{2}\right)^{5 / 2}} \\
& \times \exp \left[-\left(s_{1}+s_{2}\right)^{2} m_{e f f}^{2}-\frac{s_{1} s_{2}}{s_{1}+s_{2}} \bar{p}^{2}\right]
\end{aligned}
$$

Here we have defined the quantities,

$$
\begin{aligned}
p_{n c}^{2} & =\left(p_{1}^{(1)}\right)^{2}+\left(p_{2}^{(1)}\right)^{2} \\
m_{e f f}^{2} & =m^{2}+\frac{\left(\Delta p_{3}\right)^{2}}{\alpha^{2} p_{n c}^{2}} \\
\Delta p_{3} & =p_{3}^{(1)}-p_{3}^{(2)} \\
\bar{p}^{2} & =\left(p_{\perp}^{(1)}\right)^{2}+\frac{1}{4}\left(p_{3}^{(1)}+p_{3}^{(2)}\right)^{2} .
\end{aligned}
$$




\section{References}

[1] A. Hashimoto and S. Sethi, "Holography and string dynamics in time-dependent backgrounds," Phys. Rev. Lett. 89 (2002) 261601, hep-th/0208126.

[2] L. Dolan and C. R. Nappi, "Noncommutativity in a time-dependent background," Phys. Lett. B551 (2003) 369-377, hep-th/0210030.

[3] R. G. Cai, J. X. Lu and N. Ohta, "NCOS and D-branes in time-dependent backgrounds," Phys. Lett. B551 (2003) 178-186, hep-th/0210206.

[4] K. Dasgupta, G. Rajesh, D. Robbins, and S. Sethi, "Time-dependent warping, fluxes, and NCYM," JHEP 03 (2003) 041, hep-th/0302049.

[5] D. A. Lowe, H. Nastase, and S. Ramgoolam, "Massive IIA string theory and Matrix theory compactification," hep-th/0303173.

[6] B. L. Cerchiai, "The Seiberg-Witten map for a time-dependent background," hep-th/0304030.

[7] S. Majid and H. Ruegg, "Bicrossproduct structure of kappa Poincare group and noncommutative geometry," Phys. Lett. B334 (1994) 348-354, hep-th/9405107.

[8] J. Madore, S. Schraml, P. Schupp, and J. Wess, "Gauge theory on noncommutative spaces," Eur. Phys. J. C16 (2000) 161-167, hep-th/0001203.

[9] S. Imai and N. Sasakura, "Scalar field theories in a Lorentz-invariant three-dimensional noncommutative space-time," JHEP 09 (2000) 032, hep-th/0005178.

[10] A. Agostini, G. Amelino-Camelia and F. D'Andrea, "Hopf-algebra description of noncommutative-spacetime symmetries," hep-th/0306013.

[11] V. Kathotia, "Kontsevich's Universal Formula for Deformation Quantization and the Campbell-Baker-Hausdorff Formula, I," math.QA/9811174.

[12] T. Filk, "Divergencies in a field theory on quantum space," Phys. Lett. B376 (1996) 53-58. 
[13] S. Minwalla, M. Van Raamsdonk, and N. Seiberg, "Noncommutative perturbative dynamics," JHEP 02 (2000) 020, hep-th/9912072.

[14] M. M. Sheikh-Jabbari, "Open strings in a B-field background as electric dipoles," Phys. Lett. B455 (1999) 129-134, hep-th/9901080.

[15] D. Bigatti and L. Susskind, "Magnetic fields, branes and noncommutative geometry," Phys. Rev. D62 (2000) 066004, hep-th/9908056.

[16] L. Cornalba and R. Schiappa, "Nonassociative star product deformations for D-brane worldvolumes in curved backgrounds," Commun. Math. Phys. 225 (2002) 33-66, hep-th/0101219.

[17] J. Gomis and T. Mehen, "Space-time noncommutative field theories and unitarity," Nucl. Phys. B591 (2000) 265-276, hep-th/0005129.

[18] X. Calmet and M. Wohlgenannt, "Effective field theories on non-commutative space-time," hep-ph/0305027.

[19] O. Bertolami and L. Guisado, "Noncommutative field theory and violation of translation invariance," hep-th/0306176.

[20] J. Figueroa-O'Farrill and J. Simon, "Generalized supersymmetric fluxbranes," JHEP 12 (2001) 011, hep-th/0110170. 